\title{
Conidial surface proteome analysis reveals the highly abundant CcpA protein important for virulence of Aspergillus fumigatus
}

V. Voltersen ${ }^{1,2}$, M. G. Blango ${ }^{1}$, S. Herrmann ${ }^{3}$, F. Schmidt ${ }^{1}$, T. Heinekamp ${ }^{1,2}$, M. Strassburger ${ }^{4}$, T. Krüger ${ }^{1}$, P. Bacher ${ }^{5}$, J. Lother ${ }^{6}$, E. Weiss ${ }^{6}$, K. Hünniger ${ }^{7,8}$, H. Liu' ${ }^{9}$, P. Hortschansky ${ }^{1,2}$, A. Scheffold ${ }^{5}$, J. Löffler ${ }^{6}$, S. Krappmann ${ }^{10}$, S. Nietzsche ${ }^{11}$, O. Kurzaii,8, H. Einsele ${ }^{6}$, S. G. Filler U. Reichard ${ }^{3}$, O. Kniemeyer ${ }^{1,2}$, A. A. Brakhage ${ }^{1,2}$

'Department of Molecular and Applied Microbiology, Leibniz Institute for Natural Product Research and Infection Biology (HKI), Jena, Germany; ${ }^{2}$ Department of Microbiology and Molecular Biology, Institute of Microbiology, Friedrich Schiller University Jena, Jena, Germany; ${ }^{3}$ Institute for Medical Microbiology, University Medical Center, Göttingen, Germany; ${ }^{4}$ Transfer Group Anti-infectives, HKI, Jena, Germany ${ }^{5}$ Department of Cellular Immunology, Clinic for Rheumatology and Clinical Immunology, Charité - University Medicine, Berlin, Germany; ${ }^{6}$ Medical Clinic and Policlinic II, University Clinic Würzburg, Würzburg, Germany; 7 Institute for Hygiene and Microbiology, University of Würzburg, Würzburg, Germany; ${ }^{8}$ Septomics Research Center, HKI, Jena, Germany; ${ }^{9}$ Division of Infectious Diseases, Los Angeles Biomedical Research Institute at Harbor-UCLA Medical Center, Torrance, and Uavid Geffen School of Medicine at University of California, Los Angeles, California, USA; ${ }^{10}$ Microbiology Institute - Clinical
University of Erlangen-Nürnberg, Erlangen, Germany; ${ }^{11}$ Electron Microscopy Centre, Clinics of the Hospital, Jena, Germany

\section{Introduction}

Aspergillus fumigatus is a common airborne fungal pathogen of humans and a significant source of mortality in immunocompromised individuals. Conidia, the asexually produced spores, are the first form of $A$. fumigatus to come in contact with lung epithelial and immune cells. Thus, the composition of the conidial surface has the potential to directly influence the outcome of an $A$. fumigatus infection. Here, we aimed to identify additional conidial surface proteins which may play a role during host pathogen interaction by either hydrogen fluoridepyridine (HF/pyridine) extraction of conidial proteins or "trypsin-shaving" of surface-exposed proteins and subsequent LC-MS/MS analysis.

\section{Experimental procedures}

Conidial surface proteome

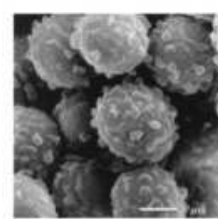

Lyophilisation
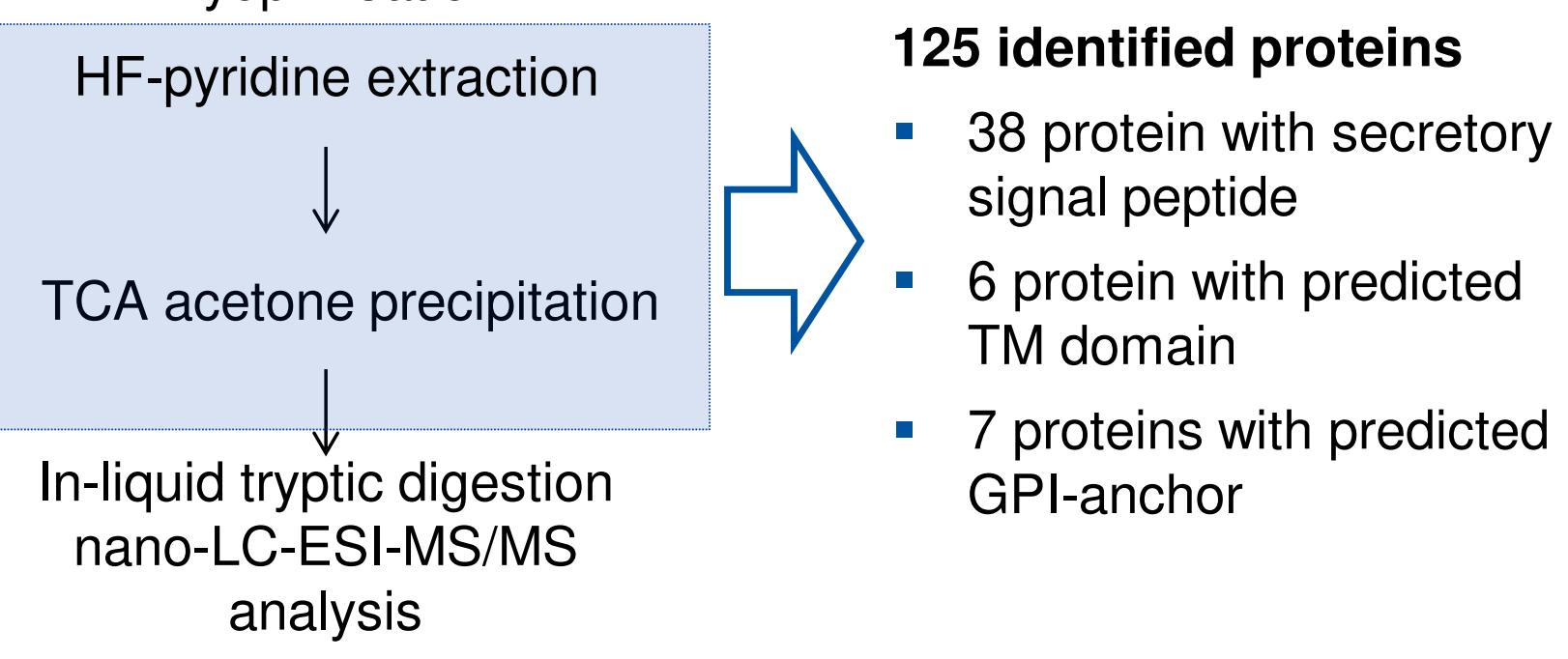

Results

The conidial cell wall protein CcpA is a highly abundant protein on the spore surface

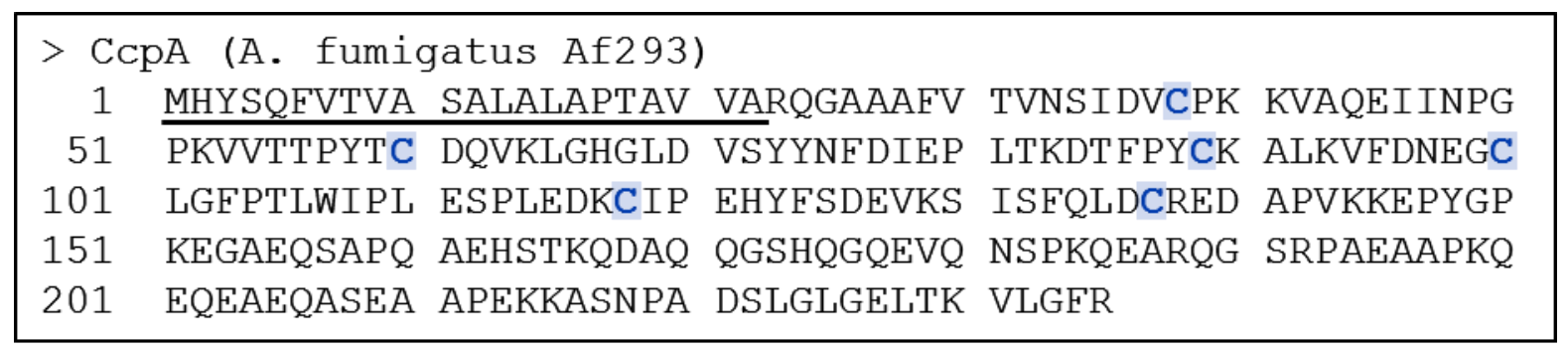

- Ccpa is an uncharacterised condial surface protein with signal peptide for secretion and 6 conserved cysteines

- It is present in Aspergilli and closely related species (Penicillium, Neosartorya)

The conidial cell wall protein CcpA is surface localised, conidia-specific, and shows developmental expression

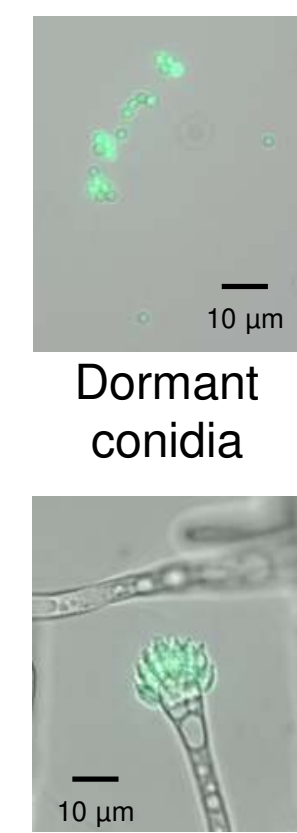

Conidiophore

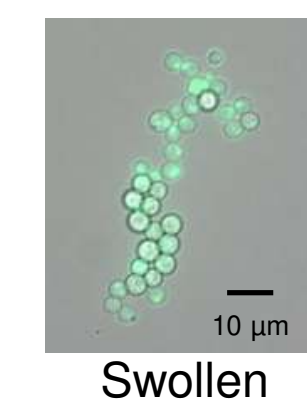

conidia

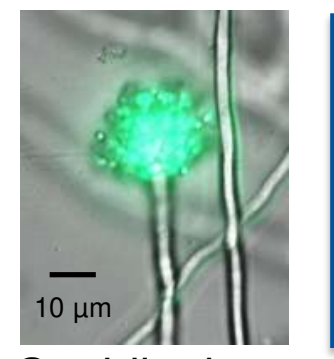

Conidiophore

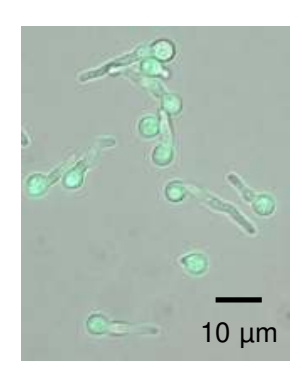

Germlings

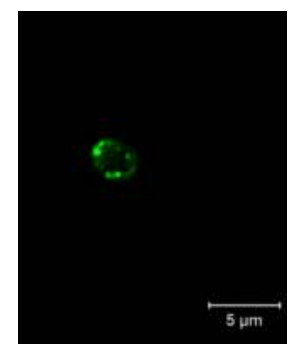

Z-stack image of dormant
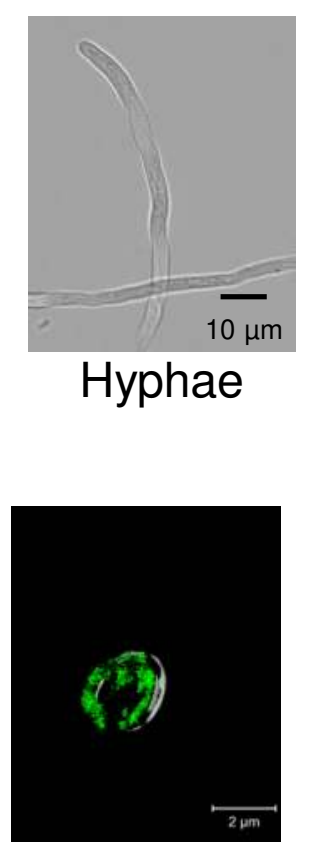

conidium

A ccpA deletion-strain ( $\triangle c c p A)$ does not show obvious growth phenotypes or changes of conidial surface properties

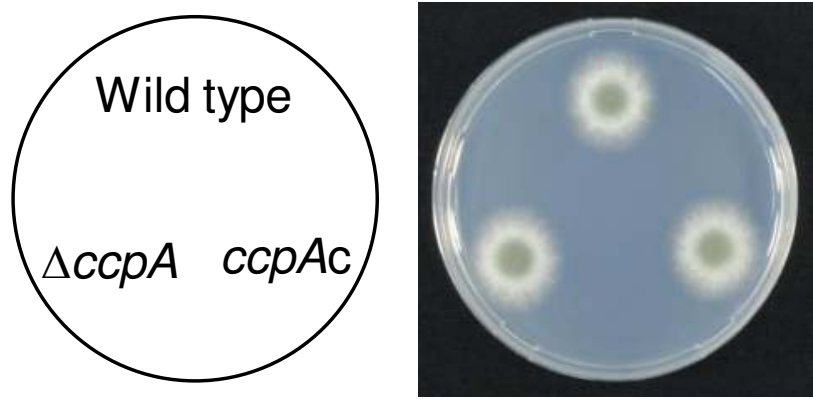

AMM medium

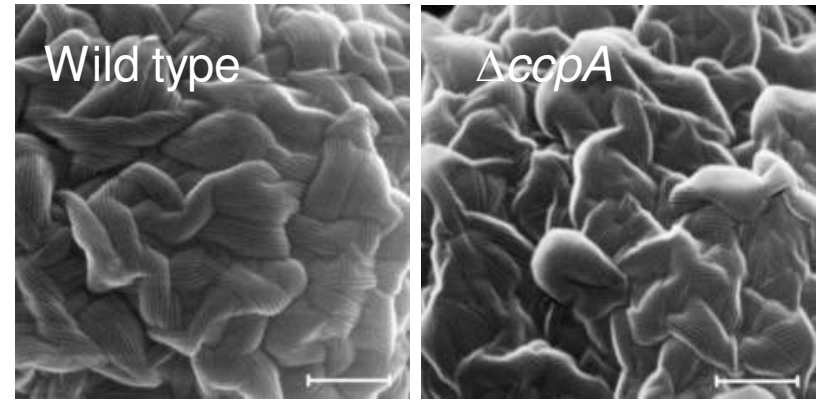

SEM image of dormant conidia (scale bar $200 \mathrm{~nm}$ )

The surface proteome of $\triangle c c p A$ is altered as revealed by surface shaving of dormant conidia with trypsin

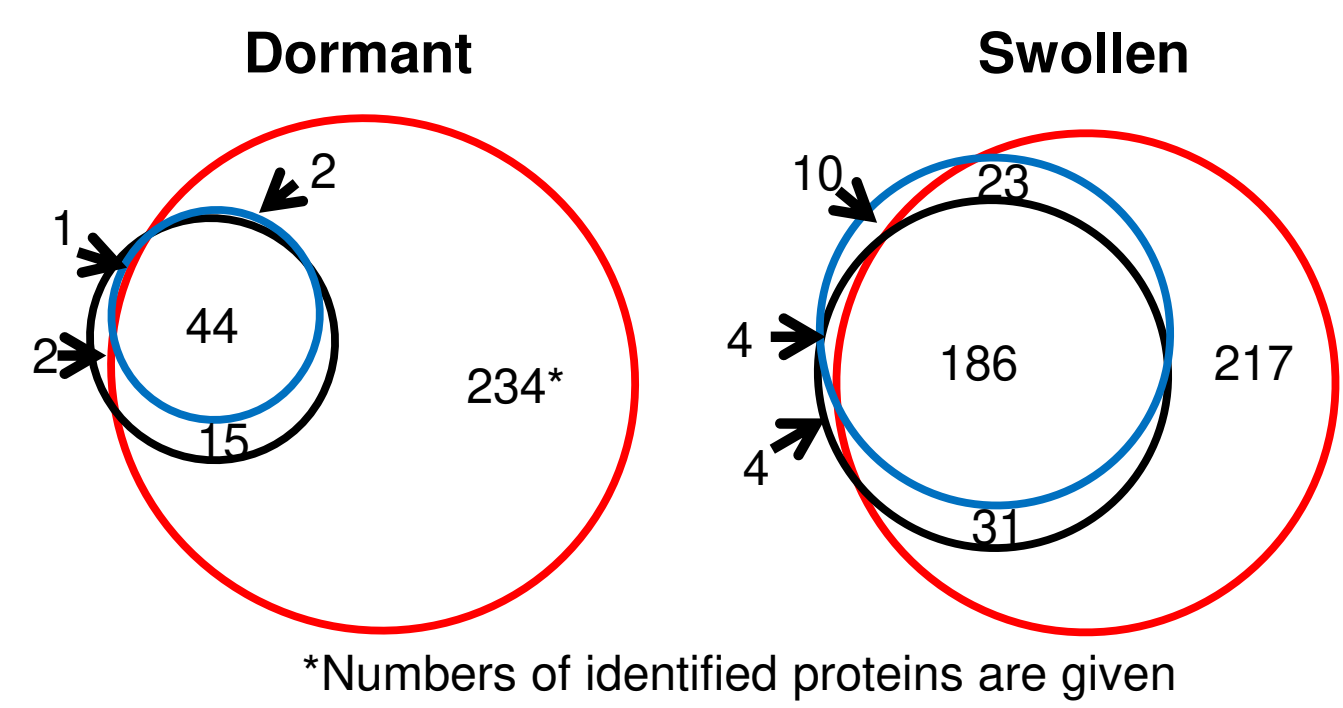

WT

$\Delta c c p A$

cсpAc

The deletion of $\operatorname{ccpA}$ leads to attenuated virulence in corticosteroidtreated mice and increased the oxidative burst in PMNs
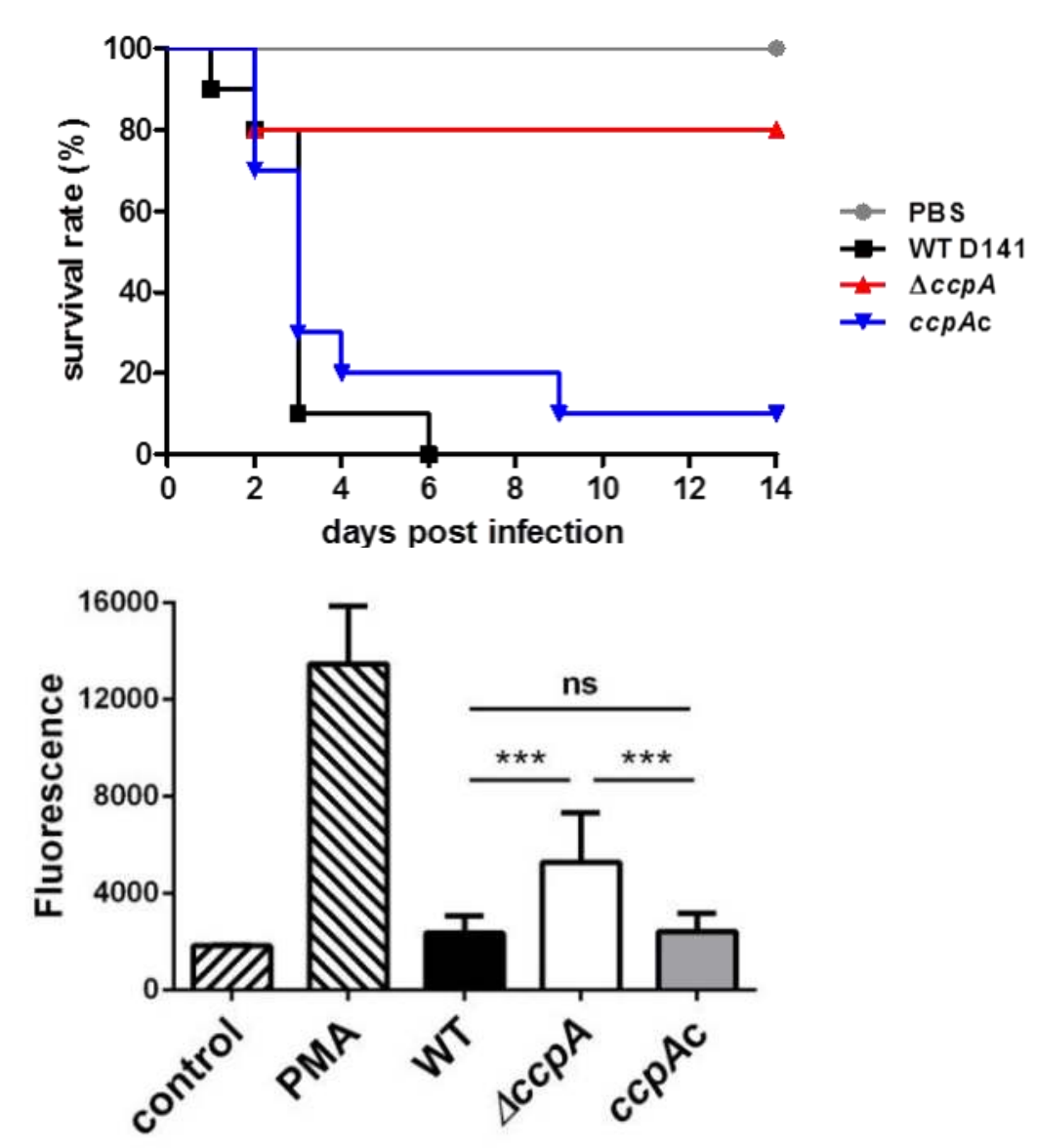

\section{Conclusions}

CcpA is a surface-localised, structural spore protein of $A$. fumigatus that is important for virulence.
Survival rates of

A. fumigatus-infected mice
Primary human PMNs were confronted for $3 \mathrm{~h}$ with swollen conidia in a DCF assay 